\title{
Hybrid Orthonormal Bernstein and Block-Pulse Method for the Solution of New System of Volterra Integro-differential Equations
}

\author{
Ahmad M. Alenezi \\ Correspondence: Department of Mathematics, The Higher Institute of Telecommunication and Navigation PAAET, \\ Shuwaikh, Kuwait
}

Received: July 6, 2020 Accepted: August 3, 2020 Online Published: August 19, 2020

doi:10.5539/jmr.v12n5p20 URL: https://doi.org/10.5539/jmr.v12n5p20

\begin{abstract}
In this article, Hybrid of Bernstein and Block-Pulse techniques (HOBM) is approaching to disband arrangement of non-linear integro-differential equations which emerge in biological model. The HOBM constringe the model of our problem into composed arrangement of non-linear necessitarian equations and this consequent variety respecting necessitarian arrangement are settled by Newton's numerical technique. Likewise, the arrangements obtained by present methodologies have been contrasted and that respecting by some current procedures accessible in writing. Some interpretative models have been discussed to show the applicability and accuracy respecting the present strategies.
\end{abstract}

Keywords: HOBM, organic species living respectively, integro-differential equations, delay integral equations

\section{Introduction}

The analysis respecting differential and integral equations is not only undeniably required for different field respecting engineering like chemical and civil engineering but likewise it is materialized to address our day to day activities. It likewise proves handy in various sectors respecting science, including electric, hydro, thermal and nuclear sectors. As we know that performance respecting arrangement Volterra Integro-differential (SVIDE) may improve in a case respecting using various technique (Marin 1996, Marin 1998, Marin 2010, Al-Jawary 2014, Yüzbaş1 and Sezer 2016). Numerous issues in designing and sciences are displayed and their frameworks. Arrangement respecting (SVIDE) emerge in logical fields, for example, Population development, Ecology, Biology, Physics, for example, electromagnetism hypothesis, one dimensional visco-flexibility and reactor elements (Marin 1996, Marin 1998, Marin 2010, Al-Jawary 2014, Yüzbaş1 and Sezer 2016). These classes respecting conditions assume a significant job in demonstrating respecting various sort respecting issues respecting building and science. This strategy is worried about the elements respecting two communicating species which are displayed as (Jerri 1999):

$$
\begin{array}{ll}
\frac{d x(t)}{d t}=x(t)\left[k k k k_{1}-\gamma_{1} y(t)-\int_{t-T_{0}}^{t} f f_{1}(t-\tau) y(\tau) d \tau\right]+ & g g_{1}(t), k k k k_{1}, \gamma_{1}>0, \\
\frac{d y(t)}{d t}=y(t)\left[-k k k k_{2}+\gamma_{2} x(t)+\int_{t-T_{0}}^{t} f f_{2}(t-\tau) x(\tau) d \tau\right]+ & g g_{2}(t), k k k k_{2}, \gamma_{2}>0,
\end{array}
$$

with the primary condition $x(0)=\alpha_{1}$, and $y(0)=\alpha_{2}$.

The up-depicting arrangement respecting Organic species living separately is concentrated in (Al-Jawary 2014). The arrangement respecting Eqs. (1)-(2) makes reference to the populace elements respecting two separate species. In this mention $x(t)$ and $y(t)$ are taken as quantities respecting independent species at time $t$. In the populace development model issues, the principal species increments, in any case, the subsequent one reduction. In the case respecting joining them together, accept that the second species $y(t)$ feed the principal species. In the pace respecting the subsequent species $\frac{d y(t)}{d t}$, there will be an expansion in the primary species, likewise which depends not just on the current species (first species) populace number $x(t)$ yet additionally on every single earlier estimation of the principal species. The issue (1) - (2) emerges when a consistent state condition is reached between these two separate species. ' $k k k k_{1}$ ' is the coefficients respecting increment and abatement respecting the main species.

' $-k k k k_{2}$ ' is the coefficients respecting increment and abatement respecting the subsequent species. 
' $\gamma_{1}$ ', ' $f f_{1}$ ' and ' $\gamma_{2}$ ', ' $f f_{2}$ ' are the realized boundaries rely upon the particular species.

' $T_{0}$ ' is limited heredity term respecting the two species.

The definite development respecting the issue is in (Jerri 1999, Khan, Vazquez-Leal et al. 2013). The specific solutions for these conditions are very much complicated even impossible in certain circumstances. Subsequently, finding the arrangements respecting the issues are exceptionally energetic either by the logical guess or numerical approximations. As respecting late, some numerical strategies endeavor to take care respecting the issue. For instance, ADM technique (Babolian and Biazar 2002), the VIM technique (Shakeri and Dehghan 2008), LMW (Yousefi 2011), SCM technique (Sahu and Ray 2016), the PPC technique (Shakourifar and Dehghan 2008), DTM and EA technique (Tari 2012). have been utilized to locate the rough arrangements respecting the arrangement respecting Eqs. (1)- (2). Likewise, CBS technique (Ramezani, Jafari et al. 2015) and BWC technique (Marin 2010)have been utilized to explain such Volterra kind vital conditions.

In this article, the above said natural model has been unraveled by HOBM technique. In general, the VIDE equation realized over interval $[a, b]$ can be transfer into the interval $[-1,1]$. HOBM has been applied to get more integral and integro-differential equations (Bhattacharya and Mandal 2008, Ordokhani and Far 2011, Maleknejad, Basirat et al. 2012, Basirat and Shahdadi 2013, Biazar, Ayati et al. 2013, Sahu and Saha Ray 2015,Mohamed R.Ali et al.). The existence strategies decrease the integro-differential equations modules to an arrangement about necessitarian equations and then this arrangement is numerically disbanded. Likewise, the comparison between existing strategies and other strategies has demonstrated in part 4. For the tables, we are affirmed that the HOBM award more precise outcomes than other strategies. Interpretative models have been talked about to exhibit the legitimacy and appropriateness regarding the proposed strategy.

This article is systematic like pursue. In part 2, we press the $H O B M$ and its matrix, Application respecting the $H O B M$ is suggested to the qualified case in part 3, part 4, manages the interpretative models which show the prospect incipiency and exactness respecting the current strategy.

\section{HOBM Functions and Some Respecting Their Properties}

In my strategies, we suspect that, $\operatorname{HOBM} i, j(x),=1,2, \ldots . M, j=0,1,2, \ldots . n$, wherever $i$ is the set respecting Block-Pulse functions, $j$ is the set respecting Orthonormal polynomials and $x$ is the standardized time, is perceived in duration $[0,1)$ as pursue:

$$
\mathrm{HOB} M_{i, j}(x)=\left\{\begin{array}{l}
B B_{j, n}(M x-i+1) \frac{i-1}{M} \leq x<\frac{i}{M} \\
\text { 0otherewise }
\end{array}\right.
$$

A set respecting Pulse functions $b l_{i}(x), i=1,2, \ldots . M$, on the interval $[0,1)$ is realized as:

$$
b l_{i}(x)=\left\{\begin{array}{lc}
1, & \frac{i-1}{M} \leq x<\frac{i}{M} \\
0, & \text { elsewhere }
\end{array}\right.
$$

The Pulse on $[0,1)$ are disjoint, so for $i, j=1,2, \ldots M$, we have $b l_{i}(x) b l_{j}(x)=\delta_{i j} b l_{i}(x)$ likewise these Tasks grasp the assets respecting altaeamud on $[0,1)$.

\subsection{Function Expansion}

The function $u(t) \in L^{2}[0,1)$ can be expanded in a hybrid orthonormal Bernstein and Block-Pulse functions

where, HOBM $i, j$ coefficients are specified by

$$
u(t)=\sum_{i=1}^{\infty} \sum_{j=0}^{\infty} c_{i j} \operatorname{HOBM} i j(t),
$$

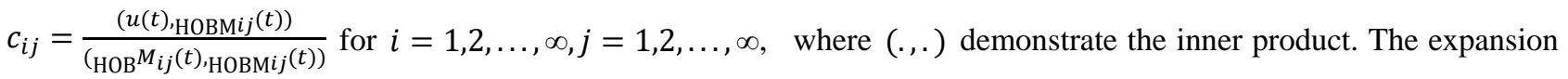

Eq. (5) have an infinite respecting part for $u(t)$. If $u(t)$ is a constant or may be truncated as a constant, then the summation in Eq. (5) can be finite after $M, n$ Parts, that is

where,

$$
u(x) \cong \sum_{i=1}^{M} \sum_{j=0}^{n} c_{i j} \operatorname{HOBM} i j(x)=C^{T} \operatorname{HOBM}^{(x)}
$$

$C=\left[c_{1,0}, c_{1,1}, \ldots, c_{M, n}\right]^{T}$,

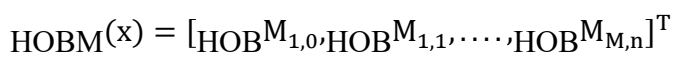

We may acquire the approximation respecting the function $k k(x, t)$ as pursue;

$\mathrm{k}(\mathrm{x}, \mathrm{t}) \approx \operatorname{HOBM}^{\mathrm{T}}(\mathrm{x}) \mathrm{K}_{\mathrm{HOBM}}(\mathrm{t})$, where $K$ is an $M(n+1) \times M(n+1)$ matrix that we have an operator of: 


$$
\mathrm{K}_{\mathrm{ij}}=\frac{\left(\mathrm{HOBM}^{\mathrm{i}}(\mathrm{x}),\left(\mathrm{k}(\mathrm{x}, \mathrm{t})_{,{ }_{\mathrm{HOBM}}}(\mathrm{t})\right)\right.}{\left(\mathrm{HOB}_{\mathrm{i}}(\mathrm{x})_{\mathrm{HOB}} \mathrm{M}_{\mathrm{i}}(\mathrm{x})\right)_{\left.\mathrm{HOB} \mathrm{M}_{\mathrm{j}}(\mathrm{t}){ }_{\text {HOBMj }}(\mathrm{t})\right)}} \text { for } i, j=1,2, \ldots, M, n
$$

\section{Application of the Techniques}

Consider the of SVIDE shown in Eqs. (1)-(2) and demonstrated the unknown of $x(t)$ and $y(t)$ by using Eq. (6) as:

$$
\begin{gathered}
x(t)=\sum_{i=1}^{M} \sum_{j=0}^{n} c_{i j} \operatorname{HOBM}_{i j}(t)=C^{T} \operatorname{HOBM}(t) \\
y(t)=\sum_{i=1}^{M} \sum_{j=0}^{n} d_{i j} \operatorname{HOBM}_{i j}(t)=D^{T} \operatorname{HOBM}(t)
\end{gathered}
$$

Here, Eqs. (1)-(2) can be compose as:

$$
\begin{gathered}
C^{T} H_{O B M^{\prime}}(t)=C^{T} H O B M(t)\left[k k_{1}-\gamma_{1} D^{T} H O B M(t)\right. \\
\left.-\int_{t-T_{0}}^{t} f f_{1}(t-\tau)\left(D^{T} H O B M(\tau)\right) d \tau\right]+g g_{1}(t), \\
D^{T} H_{O B M^{\prime}}(t)=C^{T} H O B M(t)\left[-k k_{2}+\gamma_{2} C^{T} H O B M(t)\right. \\
\left.+\int_{t-T_{0}}^{t} f f_{2}(t-\tau)\left(C^{T} H O B M(\tau)\right) d \tau\right]+g g_{2}(t),
\end{gathered}
$$

Here, we consider the integral part of Eqs. (10)-(11), and in order to utilize the Gaussian integration, we can change the period $\left[t-T_{0}, t\right]$ to $[-1,1]$ by the transformation.

$$
s=1+2\left(\frac{\tau-t}{T_{0}}\right) .
$$

Here, the integrands respecting Eqs. (10)-(11) can be compose as:

$$
\begin{gathered}
\int_{t-T_{0}}^{t} f f_{1}(t-\tau)\left(D^{T} \text { HOBM }(\tau)\right) d \tau=\frac{T_{0}}{2} \int_{-1}^{1} f f_{1}\left(-\frac{T_{0}}{2}(s-1)\right)\left(D^{T} \text { HOBM }\left(t+\frac{T_{0}}{2}(s-1)\right)\right) d s, \\
=\frac{T_{0}}{2} \sum_{j=0}^{n} w_{j} f f_{1}\left(-\frac{T_{0}}{2}\left(s_{j}-1\right)\right)\left(D^{T} \operatorname{HOBM}(t)\left(t+\frac{T_{0}}{2}\left(s_{j}-1\right)\right)\right), \\
\int_{t-T_{0}}^{t} f f_{2}(t-\tau)\left(C^{T} \text { HOBM }(\tau)\right) d \tau=\frac{T_{0}}{2} \int_{-1}^{1} f f_{2}\left(-\frac{T_{0}}{2}(s-1)\right)\left(D^{T} \text { HOBM }\left(t+\frac{T_{0}}{2}(s-1)\right)\right) d s \\
=\frac{T_{0}}{2} \sum_{j=0}^{n} w_{j} f f_{2}\left(-\frac{T_{0}}{2}\left(s_{j}-1\right)\right)\left(C^{T} \text { HOBM }\left(t+\frac{T_{0}}{2}\left(s_{j}-1\right)\right)\right),
\end{gathered}
$$

where $s_{j}, j=0,1, \ldots, n$ are the Gaussian points and $w_{j}$ are the weights and can be as;

Using Eqs. (13)-(14) in Eqs. (10)-(11), we have

$$
w_{j}=\frac{2}{\left(1-s_{j}^{2}\right)\left[\operatorname{HOBM}^{\prime}\left(s_{j}\right)\right]^{2}}
$$

$$
\begin{gathered}
C^{T} H_{O B M}(t)=C^{T} \operatorname{HOBM}(t)\left[k k_{1}-\gamma_{1} D^{T} H O B M(t)\right. \\
-\frac{T_{0}}{2} \sum_{j=0}^{n} w_{j} f f_{1}\left(-\frac{T_{0}}{2}\left(s_{j}-1\right)\right)\left(D^{T} H O B M\left(t+\frac{T_{0}}{2}\left(s_{j}-1\right)\right)\right)+g g_{1}(t) \\
D^{T} H_{O B M}(t)=D^{T} H O B M(t)\left[-k k_{2}+\gamma_{2} C^{T} H O B M(t)\right. \\
+\frac{T_{0}}{2} \sum_{j=0}^{n} w_{j} f f_{2}\left(-\frac{T_{0}}{2}\left(s_{j}-1\right)\right)\left(C^{T} H O B M(t)\left(t+\frac{T_{0}}{2}\left(s_{j}-1\right)\right)\right)+g g_{2}(t)
\end{gathered}
$$

Here, denoting the collocation points as Gauss-Legendre points $t_{i}, i=0,1, \ldots, M-1$ to the Eqs. (15)-(16), we acquire;

$$
\begin{gathered}
C^{T} \operatorname{HOBM}^{\prime}\left(t_{i}\right)=C^{T} \operatorname{HOBM}\left(t_{i}\right)\left[k k_{1}-\gamma_{1} D^{T} \operatorname{HOBM}\left(t_{i}\right)\right. \\
-\frac{T_{0}}{2} \sum_{j=0}^{n} w_{j} f f_{1}\left(-\frac{T_{0}}{2}\left(s_{j}-1\right)\right)\left(D^{T} H o B M\left(t_{i}+\frac{T_{0}}{2}\left(s_{j}-1\right)\right)\right)+g g_{1}\left(t_{i}\right) \\
D^{T} \operatorname{HOBM}^{\prime}\left(t_{i}\right)=D^{T} \operatorname{HOBM}\left(t_{i}\right)\left[-k k_{2}+\gamma_{2} C^{T} \operatorname{HOBM}\left(t_{i}\right)\right.
\end{gathered}
$$




$$
+\frac{T_{0}}{2} \sum_{j=0}^{n} w_{j} f f_{2}\left(-\frac{T_{0}}{2}\left(s_{j}-1\right)\right)\left(C^{T} H O B M\left(t_{i}+\frac{T_{0}}{2}\left(s_{j}-1\right)\right)\right)+g g_{2}\left(t_{i}\right)
$$

Again, from boundary conditions, we get

$$
C^{T} H O B M(0)=\alpha_{1}, \quad D^{T} H O B M(0)=\alpha_{2}
$$

Thus, from Eqs. (17)-(18), we acquire $M(n+1)$ non-linear equations with the unknowns as $C$ and $D$. Solving these arrangement (17)-(18) numerically, we can acquire the magnitude respecting $C$ and $D$ and hence the approximate solutions respecting the integro-differential equations (1)-(2) by using the Eqs. (8)-(9).

The underlying and cutoff esteem developing in the hypothesis regarding gases and flexibility are minor to non-straight shapes to fathom them. Due to their significant significance, various numerical and diagnostic procedures have been made for these issues because of it isn't reasonable to derive its precise arrangement by a necessitarian activity, for example, iterative numerical solvers subject to Newton's strategy. It is prominent that the hidden assessments for Newton's methodology, the course of action are basic. A procedure can be used for picking the hidden evaluations.

$$
\begin{gathered}
C_{n}=x_{n}-\frac{2 C\left(x_{n}\right) C^{\prime}\left(x_{n}\right)}{2 C^{\prime 2}\left(x_{n}\right)-C\left(x_{n}\right) C^{\prime}\left(x_{n}\right)} \\
x_{n+1}=x_{n}-\frac{\left.2\left(C\left(x_{n}\right)+C\left(C_{n}\right)\right) C^{\prime}\left(x_{n}\right)\right)}{2 C^{\prime 2}\left(x_{n}\right)-\left(C\left(x_{n}\right)+C\left(C_{n}\right)\right) C^{\prime}\left(x_{n}\right)}
\end{gathered}
$$

The nonexclusive flow outline technique is specified in Fig. 1.

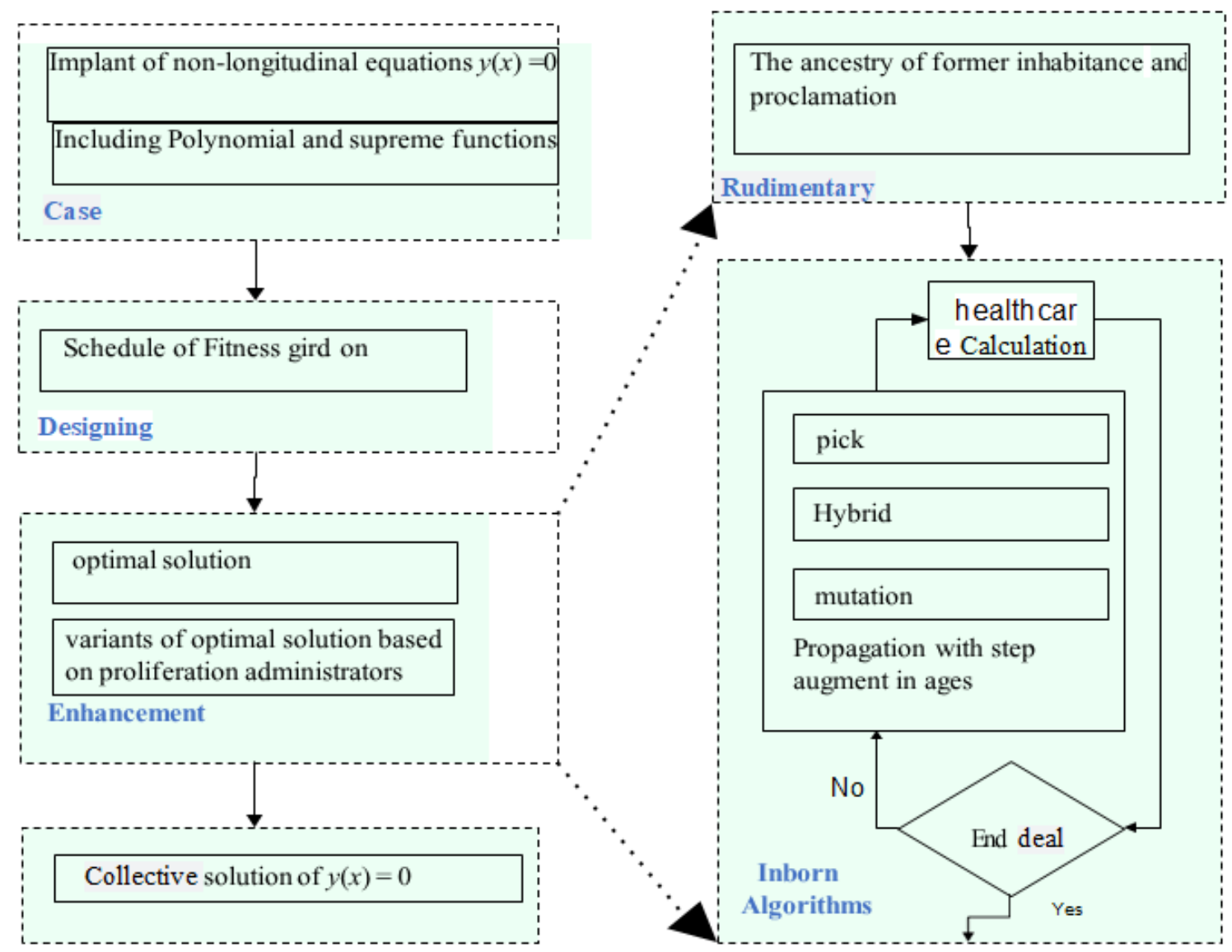

Figure1. Schematic depiction respecting the suggested methodology for detecting the solution respecting non-linear equations dependent on variations respecting germinal algorithms 


\section{Interpretative Models}

\section{Example 1 (Shakeri and Dehghan 2008)}

Consider the SVIDE realized in Eqs. (1)-(2) with;

$$
\begin{aligned}
& f f_{1}(t)=t, \quad f f_{2}(t)=t+1, \quad k k_{1}=1, \quad k k_{2}=1, \quad \gamma_{1}=\frac{1}{2}, \\
& \gamma_{2}=3 \text {, } \\
& T_{0}=\frac{1}{4} \\
& \alpha_{1}=0 \\
& \alpha_{2}=-1
\end{aligned}
$$

$g g_{1}(t)=2 t-1-\left(t^{2}-t\right)\left(1+\frac{11}{18} e^{-3 t}-\frac{1}{36} e^{\frac{3}{4}-3 t}\right)$,

and $g g_{2}(t)=\frac{1}{3072} e^{-3 t}\left(10080 t^{2}-10304 t+6275\right)$.

The specific solution respecting this model is $x(t)=t^{2}-t$ and $y(t)=-e^{-3 t}$. The numerical results acquire by HOBM for $M=4, n=1$, have compared with the solutions acquired by BPCM $(\mathrm{n}=6)$ (Sahu and Ray 2016) and VIM (Shakeri and Dehghan 2008). The correlations have been referred to in Table 1. For this issue, the got non-linear mathematical conditions framework has been disbanded numerically by Newton's strategy.

Table 1. Arbitrage respecting results obtained numerical due to example s1

\begin{tabular}{|c|c|c|c|c|c|c|}
\hline$t$ & \multicolumn{2}{|c|}{ A fundamental error (AE) for $x(t)$} & \multicolumn{3}{c|}{ AE for $y(t)$} \\
\hline & HOBM & BPCM & VIM [9] & HOBM & BPCM & VIM [9] \\
\hline 0.1 & $3.38 \times 10^{-7}$ & $1.24 \times 10^{-6}$ & $3.59 \times 10^{-7}$ & $2.24 \times 10^{-6}$ & $4.51 \times 10^{-4}$ & $1.09 \times 10^{-5}$ \\
\hline 0.2 & $1.17 \times 10^{-7}$ & $4.36 \times 10^{-6}$ & $2.66 \times 10^{-7}$ & $6.30 \times 10^{-6}$ & $4.22 \times 10^{-4}$ & $1.55 \times 10^{-5}$ \\
\hline 0.3 & $2.68 \times 10^{-7}$ & $8.83 \times 10^{-6}$ & $4.66 \times 10^{-7}$ & $6.26 \times 10^{-6}$ & $3.43 \times 10^{-4}$ & $8.22 \times 10^{-6}$ \\
\hline 0.4 & $2.48 \times 10^{-7}$ & $1.95 \times 10^{-5}$ & $1.64 \times 10^{-5}$ & $2.43 \times 10^{-5}$ & $2.89 \times 10^{-4}$ & $9.26 \times 10^{-4}$ \\
\hline 0.5 & $2.52 \times 10^{-7}$ & $2.50 \times 10^{-5}$ & $6.93 \times 10^{-5}$ & $5.37 \times 10^{-6}$ & $2.44 \times 10^{-4}$ & $3.95 \times 10^{-4}$ \\
\hline 0.6 & $2.17 \times 10^{-7}$ & $3.02 \times 10^{-5}$ & $1.73 \times 10^{-4}$ & $2.40 \times 10^{-5}$ & $1.99 \times 10^{-4}$ & $9.37 \times 10^{-3}$ \\
\hline 0.7 & $2.23 \times 10^{-7}$ & $3.52 \times 10^{-5}$ & $3.23 \times 10^{-4}$ & $1.32 \times 10^{-5}$ & $1.65 \times 10^{-4}$ & $1.62 \times 10^{-3}$ \\
\hline 0.8 & $4.30 \times 10^{-7}$ & $4.01 \times 10^{-5}$ & $4.92 \times 10^{-4}$ & $1.27 \times 10^{-6}$ & $1.44 \times 10^{-4}$ & $2.26 \times 10^{-3}$ \\
\hline 0.9 & $5.48 \times 10^{-7}$ & $4.48 \times 10^{-5}$ & $6.41 \times 10^{-4}$ & $2.34 \times 10^{-5}$ & $1.20 \times 10^{-4}$ & $2.63 \times 10^{-3}$ \\
\hline
\end{tabular}

\section{Example 2}

The example respecting Organic species living respectively with;

$$
\begin{array}{lllll}
f f_{1}(t)=t, & f f_{2}(t)=t+1, & k k_{1}=0.02, & k k_{2}=0.01, & \gamma_{1}=0.01, \\
\gamma_{2}=0.01, & T_{0}=0.1, & \alpha_{1}=10, & \alpha_{2}=10, & g g_{1}(t)=0,
\end{array}
$$

and $\quad g g_{2}(t)=0$.

The solutions got by the strategies $(\operatorname{HOBM}(\mathrm{M}=8, \mathrm{n}=7))$ have been contrasted by the solutions acquired by Bernstein polynomial collocation technique BPCM $(\mathrm{n}=6)$ ) [21], ADM [8] and HPM [20] for the same magnitude of $t$, and the similarities are characterized in Table 2. Due to this module, the gained non-linear necessitarian equations arrangement acquired by Newton's technique numerically.

Table 2. Resemblance respecting numerical arrangement due to example 2

\begin{tabular}{|c|c|c|c|c|c|c|c|c|}
\hline$t$ & \multicolumn{4}{|c|}{$x(t)$} & \multicolumn{4}{c|}{$y(t)$} \\
\hline & HOBM & BPCM & ADM & HPM & HOBM & BPCM & ADM & HPM \\
\hline 0.1 & 9.01399 & 9.01497 & 8.968 & 8.9668 & 11.0913 & 11.0902 & 11.162 & 11.0416 \\
\hline 0.3 & 7.07823 & 7.07823 & 6.905 & 6.8911 & 13.2279 & 13.2279 & 13.485 & 13.1250 \\
\hline 0.5 & 7.07726 & 5.31893 & 4.842 & 4.8030 & 15.16 & 15.159 & 15.808 & 15.2082 \\
\hline 0.7 & 5.31809 & 3.84465 & 2.780 & 2.7023 & 16.7701 & 16.7692 & 18.131 & 17.2916 \\
\hline
\end{tabular}

From the table 1,2 we can show that HOBM demonstrate more accurate and greater solutions than other strategies such as (BPCM, HPM, and ADM) Table 2 cites that solutions acquired by HOBM and BPCM have the same quite satisfactorily and these solutions are likewise the same as the other technique solutions. 


\section{Conclusion}

System respecting integro-differential modules that can be appeared in the Biological frameworks which are disbanded numerically utilizing HOBM technique and the acquired solutions compared with the solutions acquired by other strategies. The model issue is changed over into non-linear logarithmic equations. The group equations are disbanded using Newton-Raphson techniques. The HOBM solutions are compared with an existing technique. The interpretative models have been incorporated to exhibit the legitimacy and relevance respecting the proposed strategies. These models additionally show the exactness and productivity respecting the current strategies.

\section{References}

Al-Jawary, M. (2014). Approximate solution respecting a model describing biological species living together using a new iterative method. International Journal of Applied Mathematics Research, 3(4), 518.

Ali, M. R., \& Ma, W. X. (2020). New exact solutions of Bratu Gelfand model in two dimensions using Lie symmetry analysis. Chinese Journal of Physics.

Ali, M. R., \& Baleanu, D. (2019). Haar wavelets scheme for solving the unsteady gas flow in four-dimensional. Thermal Science, (00), 292-292.

Ali, M. R., Hadhoud, A. R., \& Srivastava, H. M. (2019). Solution of fractional Volterra-Fredholm integro-differential equations under mixed boundary conditions by using the HOBW method. Advances in Difference Equations, 2019(1), 115.

Babolian, E., \& Biazar, J. (2002). Solving the problem of biological species living together by Adomian decomposition method. Applied Mathematics and computation, 129(2-3), 339-343. https://doi.org/10.1016/S0096-3003(01)00043-1

Basirat, B., \& Shahdadi, M. A. (2013). Numerical solution of non-linear integro-differential equations with initial conditions by Bernstein operational matrix of derivative. International Journal of Modern Nonlinear Theory and Application, 02(02), 141-149.

Bhattacharya, S., \& Mandal, B. N. (2008). Numerical solution of a singular integro-differential equation. Applied Mathematics and Computation, 195(1), 346-350. https://doi.org/10.1016/j.amc.2007.04.103

Biazar, J., Ayati, Z., \& Partovi, M. (2013). Homotopy perturbation method for biological species living together. International Journal of Applied, 2(1), 44-48.

Dehghan, M., \& Shakeri, F. (2007). Solution of a partial differential equation subject to temperature overspecification by He's homotopy perturbation method. Physica Scripta, 75(6), 778.

Khan, Y., Vazquez-Leal, H., \& Wu, Q. (2013). An efficient iterated method for mathematical biology model. Neural Computing and Applications, 23(3-4), 677-682.

Kumar, R., Miglani, A., \& Rani, R. (2018). Generalized Two Temperatures Thermoelasticity of Micropolar Porous Circular Plate with Three Phase Lag Model. Journal of Mechanics, 34(6), 779-789.

Othman, M. I. A., \& Atwa, S. Y. (2012). Response of micropolar thermoelastic solid with voids due to various sources under Green Naghdi theory. Acta Mechanica Solida Sinica, 25(2), 197-209.

Maleknejad, K., Basirat, B., \& Hashemizadeh, E. (2012). A Bernstein operational matrix approach for solving a system of high order linear Volterra-Fredholm integro-differential equations. Mathematical and Computer Modelling, 55(3-4), 1363-1372.

Marin, M. (2010). A partition of energy in thermoelasticity of microstretch bodies. Nonlinear Analysis: Real World Applications, 11(4), 2436-2447.

Mirzaee, F., \& Samadyar, N. (2017). Application of orthonormal Bernstein polynomials to construct a efficient scheme for solving fractional stochastic integro-differential equation. Optik, 132, 262-273.

Ramezani, M., Jafari, H., Johnston, S. J., \& Baleanu, D. (2015). Complex b-spline collocation method for solving weakly singular volterra integral equations of the second kind. Miskolc Mathematical Notes, 16(2), 1091-1103.

Sahu, P., \& Saha, R. S. (2015). A new numerical approach for the solution of non-linear Fredholm integral equations system of second kind by using Bernstein collocation method. Mathematical Methods in the Applied Sciences 38(2), 274-280. https://doi.org/10.1002/mma.3067

Sahu, P. K., \& Ray, S. S. (2016). Legendre spectral collocation method for the solution of the model describing biological species living together. Journal of Computational and Applied Mathematics, 296, 47-55. 
Shakourifar, M., \& Dehghan, M. (2008). On the numerical solution of nonlinear systems of Volterra integro-differential equations with delay arguments. Computing, 82(4), 241.

Tari, A. (2012). The differential transform method for solving the model describing biological species living together.

Yousefi, S. A. (2011). Numerical solution of a model describing biological species living together by using Legendre multiwavelet method. International Journal of Nonlinear Science, 11(1), 109-113.

Yasir, K., Héctor, V., \& Wu, Q. (2012). An efficient iterated method for mathematical biology model, Neural Computing and Applications.

\section{Copyrights}

Copyright for this article is retained by the author(s), with first publication rights granted to the journal.

This is an open-access article distributed under the terms and conditions of the Creative Commons Attribution license (http://creativecommons.org/licenses/by/4.0/). 